\title{
Transforming vaccine development
}

4

Steve Black ${ }^{1}$, David E. Bloom ${ }^{2}$, David C. Kaslow ${ }^{3}$, Simone Pecetta $^{4}$ and Rino Rappuoli ${ }^{4,5^{*}}$

${ }^{1}$ Cincinnati Children's Hospital, Cincinnati, OH 45229, USA

${ }^{2}$ Harvard T.H. Chan School of Public Health, Harvard University, Boston MA 02115, USA

${ }^{3}$ PATH, Seattle WA 98121, USA

${ }^{4}$ GSK, 53100 Siena, Italy

${ }^{5}$ Imperial College London, London SW7 2AZ, UK

*corresponding author: rino.r.rappuoli@gsk.com

Keywords: COVID-19; vaccine; vaccine development; vaccine discovery; systems biology; machine learning; platform technologies; adjuvants; smart clinical trials; human genetics; regulatory convergence; real world evidence; vaccines safety

\section{Abstract}

The urgency to develop vaccines against Covid-19 is putting pressure on the long and expensive development timelines which are normally required for development of lifesaving vaccines. There is a unique opportunity to take advantage of new technologies, smart and flexible design of clinical trials, and evolving regulatory science to speed up vaccine development against Covid-19 and transform vaccine development altogether.

\section{Introduction}

Vaccines have had a tremendous positive effect on public health and well-being. Today, the desperate need for vaccines to protect people from the pandemic caused by the 2019 novel coronavirus (SARSCoV-2, cause of COVID-19 disease), is a strong reminder for the value of vaccines, and of the importance of being able to develop and scale-up their use in a timely manner. Indeed, vaccines can not only save lives but also prevent the impact of infectious diseases on the global economy that in the case of Covid-19 has already killed more than 250,000 people, caused the unemployment of 25 million people in the USA alone and a loss of several trillion dollars to the global economy.

In addition to the immediate need for Covid-19, vaccines have the potential to address many other pathogens such as respiratory syncytial virus, cytomegalovirus, human immunodeficiency virus (HIV), and pandemic and seasonal influenza, as well as other viral emerging infections such as Ebola, Nipah, Zika, dengue, Lassa, and Middle East Respiratory Syndrome. Vaccines also offer the potential to address the mounting threat of antimicrobial resistance by targeting tuberculosis, Salmonella spp, Shigella spp, Staphylococcus aureus, Neisseria gonorrhoeae, carbapenem-resistant Enterobacteriaceae, and other resistant bacteria that have been prioritized by the World Health 
39 Organization (WHO); they can also target other bacteria, viruses, funguses, and parasites that are 40 frequently treated (whether appropriately or inappropriately) with antimicrobials, thereby promoting 41 resistance in colonizing pathogens. If all or many of these opportunities for vaccine development are 42 brought to fruition, the resulting impact on mortality, quality of life, and poverty could be 43 transformative (1).

44 Usually 15-20 years are required between the initial scientific discovery and vaccine licensure and policy recommendation (Fig. 1). Indeed, the trend over the past 30 years, for national regulatory agencies, policy-making bodies, and manufacturers has been to require a growing number of clinical studies of increasing size and complexity before licensure and recommendation; more recently, yet additional post-licensure studies have been required before broad recommendation of vaccine use. As a result, promising vaccine candidates that have established clinical proof-of-concept and demonstrated the potential for significant public health impact failed to reach people who needed them in a timely manner, leaving many unnecessarily at risk for years. Even in the case of a Public Health Emergency of International Concern (PHEIC), such as Ebola, it took 5 years, from 2014 to 2019 , to get a licensed vaccine. The long timelines and the huge investments required discourage vaccine manufacturers (and their actual and potential shareholders) from investing in innovation.

Today, the awareness that the Covid-19 pandemic will only be controlled when vaccines are made broadly available for all people, is an incredible challenge to the classical vaccine development timelines. Below we provide a list of possible opportunities that can be considered to fast track development of vaccines for Covid-19, but also transform forever the way we develop vaccines.

\section{Discovery and early development.}

60 The first opportunity comes in the discovery and early development phases described in Figure 1. 61 Here a number of technological advances now make it possible to accelerate this dramatically. For 62 instance, the availability of the genomic sequence makes possible to generate synthetic genes and 63 nucleic acid vaccines (based on DNA or RNA) for laboratory testing in a week (2). In addition, the 64 availability of the atomic structure allows the structure-based design of optimized antigens within the 65 same timeframe (3). Optimized, synthetic genes can also be spliced into viral vectors (such as human or chimpanzee Adenoviruses, Measles, Vesicular Stomatitis Virus, and others) to rapidly produce a vaccine or used to engineer mammalian, insect or plant cells to produce recombinant proteins that can be used alone or with adjuvants in a vaccine. All these acceleration opportunities that have been used to fast track Covid-19 vaccines during the last few months and can also be used for other vaccines. High-throughput analysis of genomes from bacteria, viruses, and parasites isolated worldwide can provide early knowledge of the global epidemiology of diseases and the same time, combined with structural biology, and human monoclonal antibodies, allow to identify protective antigens and epitopes through a process known as "reverse vaccinology." 


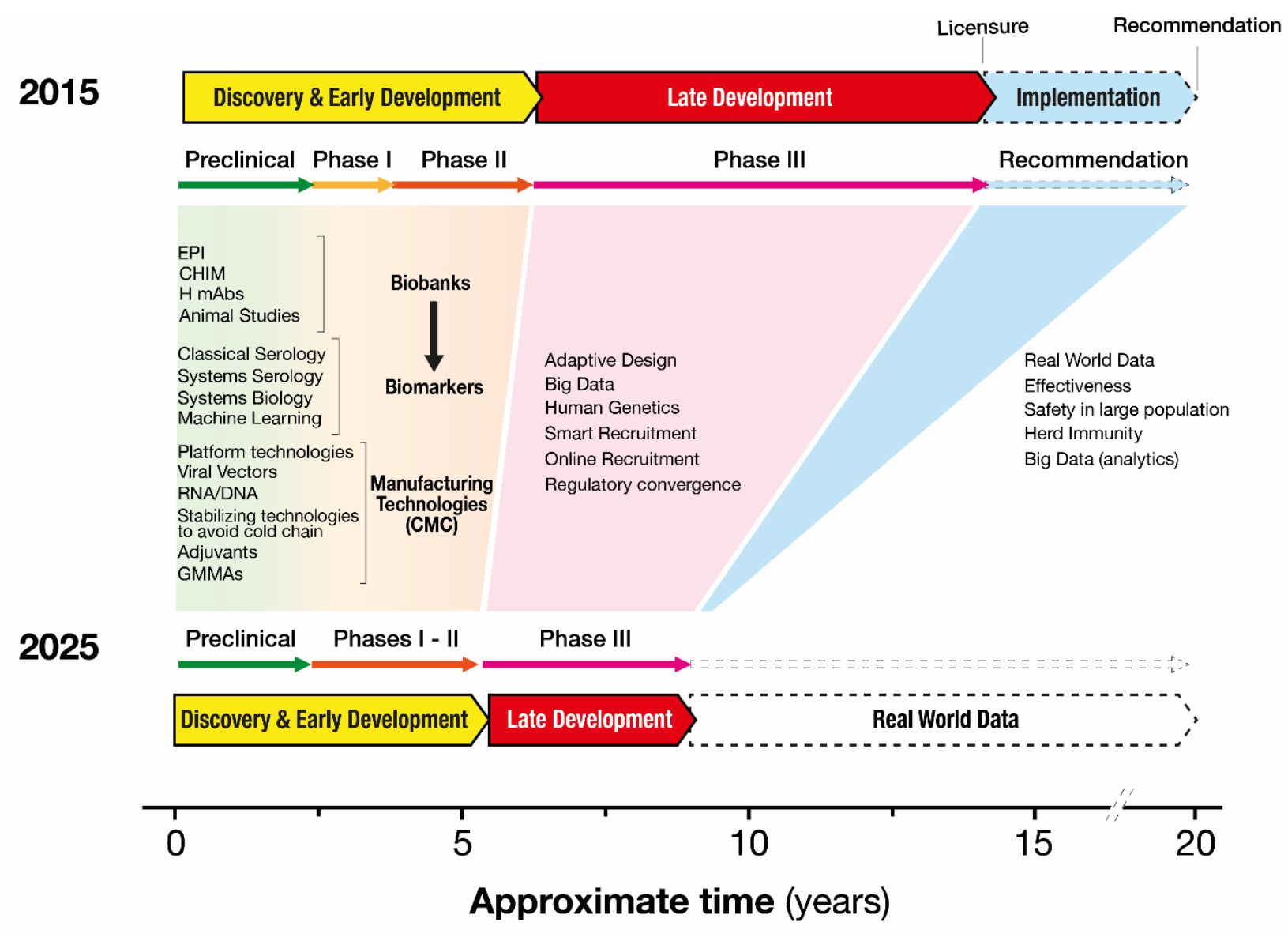

Figure 1. Innovative scientific approaches to accelerate vaccine development, divided by discovery and early development (yellow), late development (red), and implementation (blue). Phase I, Phase II, and Phase III indicate Phase I clinical trials, Phase II clinical trials, and Phase III clinical trials, respectively. EPI = epidemiological studies; $\mathrm{CHIM}=$ controlled human infection model studies; $\mathrm{H}$ mAbs = human monoclonal antibodies; GMMAs = general modules for membrane antigens; $\mathrm{CMC}=$ chemistry and manufacturing controls.

The discovery and early development phase (containing preclinical, Phase I and Phase II in Figure 1) can also be used to address many of the questions that until today have been addressed later in development using large clinical studies. For instance, in the case of Covid-19 it is imperative to obtain biobanks of blood and possibly tissue samples collected from natural disease, from appropriate animal models and possibly from controlled human infection challenge models (CHIMs) to identify biomarkers that can support the regulatory decision-making processes. In the case of Covid-19 the most pressing need is to identify the virus neutralizing titer that confers protection. For Group B streptococcal infection, epidemiological studies analyzing the immune status of people who become ill compared with that of those who remained healthy in spite of being in the same environment revealed that high antibody levels in pregnant women correlated with protection from Group B Streptococcus disease in their newborns (4). CHIM studies, presently in discussion for Covid-19 (5), are being developed for an increasing number of diseases, and in the case of cholera, results from a CHIM provided pivotal data for vaccine licensure (6).

Biobanks of sera and cells from the types of studies mentioned above can also be used to perform traditional serology studies such as viral neutralization and bactericidal or opsonophagocytic activity 
against bacteria. They can also be a source of materials for systems serology and systems biology, which offer powerful new tools to generate hypotheses for the identification of new biomarkers. Systems biology approaches, which use multi-omics data sets such as transcriptomics, metabolomics, high dimensional flow cytometry to discover mechanisms and correlates of vaccine efficacy $(7,8)$, including systems serology techniques to assess serological responses, have been used to find correlates of protection and/or risks for the malaria RTS,S vaccine and for the HIV RV144 vaccine candidate. Both correlates can be used as biomarkers to predict vaccine efficacy $(19,20)$. Systems biology is also becoming increasingly important to predict safety and protection in vaccinated people (9).

Finally, the recent innovation of high-throughput cloning of human B cells from convalescent or vaccinated people provides an additional unprecedented means of both accelerating vaccine development and developing therapeutic antibodies at the same time. For instance, the human monoclonals recently recommended by the WHO for treatment of Ebola (10), which were developed in less than five years, have also provided definitive evidence that antibodies alone can protect people from disease and that the protective level of the antibodies can be used as a biomarker to license future vaccines. Indeed, the clinical development of human monoclonals anticipates many of the scientific questions such as correlates of protection that can inform clinical and regulatory questions and endpoints that otherwise would need to be developed later for vaccine development (11). Human monoclonal antibodies derived from memory B cells of Covid-19 convalescent people are being developed (12), and are likely going to be among the first drugs to approach approval for Covid-19 therapy and prevention.

In addition, the overall vaccine development timeline can be accelerated by using vaccine technologies that can be applied to manufacture different vaccines (platform technologies), so that the technical, regulatory and manufacturing experience and capacity can be applied to new vaccines. In the case of Covid-19, RNA and vector-based vaccines, already in clinical development for many other targets, allowed developers to reach phase I clinical studies in record time of 65 days $^{1}$. In addition, the long experience with safe and effective protein-based vaccines and adjuvants is likely going to make it easier to develop vaccines based on the recombinant Covid-19 spike protein. Another platform technology presently in development are General Modules for Membrane Antigens (GMMAs) for bacterial vaccines (13).

\section{Late Development.}

The second opportunity comes in the design of clinical trials and late development (Fig. 1). The classical pathway of doing Phase I clinical studies, followed by increasingly large Phase II and then Phase III studies can be replaced by smart and flexible trials by implementing adaptive trial designs where the information gained throughout a trial can be used to modify its design as the trial is being executed. This approach, already successfully implemented for drug trials, requires that information derived from biomarkers becomes available quickly during trial execution. Usually predictive biomarkers are not routinely available in early vaccine development, however today we should be able to identify them and make the information available in real time, by modifying the design of the trials and using new technologies, especially systems serology and systems biology, supported by

\footnotetext{
${ }^{1}$ Source: https://www.modernatx.com/modernas-work-potential-vaccine-against-covid-19 (accessed on May $6^{\text {th }}, 2020$ )
} 
137 evidence from relevant animal models when those exist. Trial enrollment should also be accelerated. 138 In the case of Covid-19 internet-based recruitment is easily providing a long list of volunteers eager 139 to get protection against this pandemic virus ${ }^{1}$. However, similar tools could be used for many other 140 vaccines thanks to the increased knowledge of human genetics and databases that identify people 141 susceptible to a given disease or at risk of side effects. For instance, the U.K. biobank has been used 142 to identify individuals who are susceptible to tuberculosis, while particular genotypes have been 143 shown to predict susceptibility to severe adverse reactions to measles, mumps, and rubella (MMR) 144 and yellow fever live-attenuated virus vaccines (14).

145 The ability to design trials differently and to collect more data from each individual could have a 146 profound influence on the late development stage by enabling smaller and shorter trials that provide 147 the necessary information for licensure and recommendation; a more comprehensive assessment of 148 safety and efficacy, which requires upwards of many tens of thousands of people because of the 149 expected rarity of certain adverse events, could then be quickly and accurately made after vaccine 150 implementation. Employment of large clinical databases and statistical techniques, such as 151 maximized sequential probability ratio testing (15), could further accelerate and enhance the process 152 of post-licensure safety and effectiveness assessment.

153 It is not yet clear what is the optimum approach to acquire sufficient evidence of efficacy to license 154 and recommend Covid-19 vaccines. The traditional approach to pre-licensure evaluation of efficacy 155 and safety is based upon the premise that very large phase III studies are the best way to generate 156 objective and robust information on vaccines' safety and efficacy. While double-blind placebo157 controlled clinical studies are the conventional gold standard and that they should be used in all 158 situations in which they are appropriate, in some instances, especially thanks to the new technologies, 159 alternative approaches can provide results that are at least as reliable, and faster. Below are some 160 examples where alternative approaches were used because double blind-placebo controlled trials were 161 not possible or required unacceptable time or investments. In the case for Ebola, ring vaccination was 162 used to acquire the evidence for vaccine licensure (16), and in the case of cholera CHIM studies were 163 used (6). In other circumstances correlates of protection have been used for vaccine licensure and 164 recommendation and the impact of vaccination has been collected later by real world evidence. 165 Examples that this approach can greatly accelerate the development process and provide a more 166 realistic safety assessment derive from the experiences of introducing meningococcus $\mathrm{C}$ (MenC) and 167 meningococcus B (MenB) vaccinations in the United Kingdom. In 1999, the MenC vaccine was 168 licensed based on safety and immunogenicity data from phase II clinical data and then used to 169 vaccinate the entire U.K. population aged two months to 18 years. Serum bactericidal assay, which 170 was broadly accepted as a regulatory biomarker for meningococcal polysaccharide vaccines but not 171 previously used for conjugate vaccines, was the basis for licensure. The real-world evidence showed 172 that the disease had virtually disappeared from the country just one year after introduction and that 173 the herd immunity induced by vaccination had reduced the circulation of this serogroup in the entire 174 country, thus protecting nonvaccinated cohorts (17). Unfortunately, this demonstration of the value 175 of herd immunity had an unintended consequence, as health economists and recommending bodies 176 have since requested demonstration of herd effects before issuing broad recommendations resulting 177 in long delays. Even more recently, the value of rapid vaccine implementation followed by real-

${ }^{1}$ Source: $\underline{\text { https://covid19vaccinetrial.co.uk/ }}$ (accessed on May $6^{\text {th }}, 2020$ ) 
178 world evidence was again confirmed in the U.K. through the introduction and post-licensure testing 179 of the MenB vaccine (18). Following licensure, the vaccine was introduced in the national 180 immunization program in 2015. After three years and the administration of millions of doses, the 181 study reported a $75 \%$ reduction in disease in the vaccinated population and provided robust data about 182 the general safety of the vaccine.

\section{Health economics.}

184 The third opportunity resides in the health economics behind the regulatory approaches required for 185 licensure and recommendation of vaccines. These considerations clearly depend on the risk/benefit 186 balance. This balance is illustrated in Figure 2. As shown, while a moderate risk aversion results in 187 a great increase of public health benefit, an excessive risk aversion rapidly decreases the benefit. In 188 the case of Covid-19 the pressure is high to move fast. This justifies an appropriate but not excessive 189 risk aversion because the loss of lives and the economic impact of every additional day needed to 190 make the vaccine available are self-evident. However, during routine vaccine development many of 191 these factors are often overlooked and while it is easy to include in the analysis the costs of 192 implementing vaccine programs, it is also easy to forget the opportunity costs associated with the 193 large expenditure of time and resources necessitated by very large trials, and the cost in terms of lives 194 and productivity lost in the target population because of the length of time taken to develop and 195 introduce a new vaccine. The consequence is that a traditional vaccine development timeline requires 196 15-20 years and a cost of US\$1 billion, which clearly impact how many vaccines a pharmaceutical 197 company can develop, favor development of vaccines that target high-income populations, and make 198 the development and implementation of novel vaccines for public immunization programs in low199 and middle-income countries (LMICs) either unattractive or unsustainable (19). The need to speed 200 up vaccine development could be increased by including these data in a more comprehensive health 201 economic analysis. As an example, in Table 1 we report the theoretical lives that would be saved for 202 a few vaccines in the ideal situation in which vaccines were introduced after licensure in all target 203 geographies and assuming immediate availability of vaccines for all target populations. We estimate, 204 for example, that every year the introduction of a malaria vaccine is delayed would result in up to $205234,000(95 \%$ CI $=65,934-328,042)$ lives lost in Sub-Saharan Africa alone (20, 21). A more 206 conservative estimate from a modeling study comprised of four models still indicated the impact of 207 RTS,S to be between 16,933-34,400 averted deaths per year (22) assuming partial coverage; which, 208 over the multi-year delay for this vaccine, is still a substantial number of lives. Similarly, the delay 209 in the use of a one-dose schedule for human papillomavirus (HPV) vaccination in high-risk areas in 210 Africa has resulted in an estimated 8,991 lives lost per year $(23,24)$, while the impact of the delay of 211 a two-dose vaccine in LMICs would be up to 151,687 lives lost per year. Furthermore, recent 212 advances in tuberculosis vaccine development suggest that almost 1 million deaths per year could be 213 saved by early introduction of a vaccine worldwide. 


\section{Public Health Benefit versus Risk Aversion}

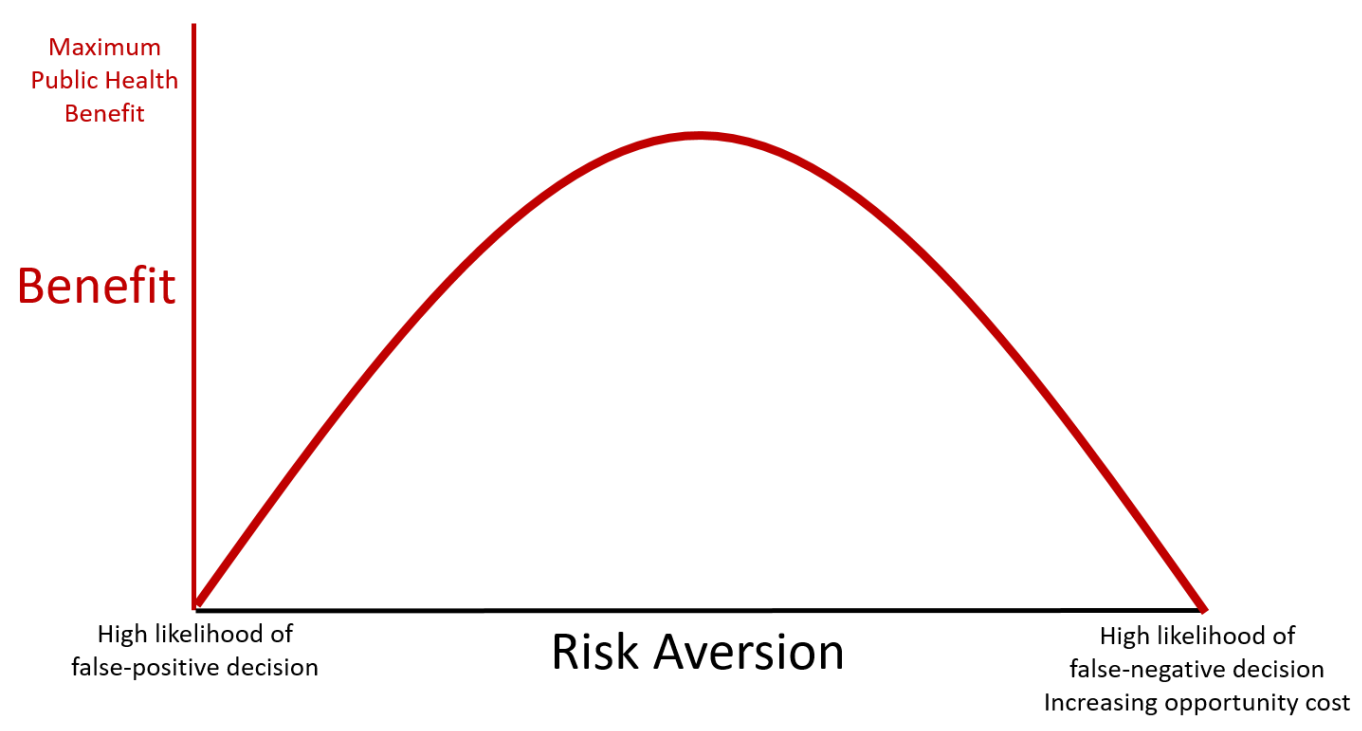

Adapted from: Eichler, H-G et. al. Nature Reviews Drug Discovery doi:10.1038/nrd4129

Figure 2. Net public health benefits versus risk aversion in product development. The relationship between risk aversion by regulators on the $\mathrm{x}$-axis, and the expected net public health benefits on the y-axis. The desire to minimize the likelihood of accepting products with unpredicted product-induced adverse effects drives the request for larger and larger safety datasets. By continuing to move to the right and beyond a 'sweet spot' of maximum efficiency, increased risk aversion or requests for more data are anticipated to result in diminishing net public health gains from product research and development. The unintended consequences are false-negative decision to deny licensure or restrict a product when actual use would result in more good than harm and increased opportunity costs (25).

\section{Regulatory environment.}

Regulatory agencies are generally and increasingly open to embracing innovation and accepting evidence from new technologies, provided that the innovation has been properly validated. In fact, some agencies are already moving toward implementation of several of the tools and techniques mentioned here in other areas of pharmaceutical development, especially with respect to cancer drugs (26, 27). Recent agency-sponsored workshops (for example, the U.S. FDA's Identification and Use of Biomarkers to Advance Development of Preventive Vaccines: Public Workshop ${ }^{1}$ ) have provided insights and written guidelines on how to implement adaptive trial design, how to use biomarkers, and how to use real-world data for the development of certain therapies (28-30).

In the case of Covid-19 the major regulatory agencies are having a good dialog and overall a common and collaborative approach, however this is not the case in most circumstances. Establishing an agreement between regulatory agencies from around the world on the evidence necessary for vaccine licensure is of paramount importance to facilitate and accelerate development and introduction of new vaccines globally. This regulatory convergence would avoid unnecessary duplications of clinical trials and of regulatory information required for vaccine licensure in different countries.

\footnotetext{
${ }^{1}$ Source: https://www.fda.gov/vaccines-blood-biologics/workshops-meetings-conferences-biologics/identification-anduse-biomarkers-advance-development-preventive-vaccines-public-workshop-09162019 (accessed on May 6 ${ }^{\text {th }}, 2020$ )
} 
238 Development of master protocols, providing single "umbrella trial designs" that can be applied to 239 multiple vaccines, with pre-specified methods for planning or modifying the sample size, dropping 240 an arm, or other adaptive strategies; and endorsed by the different relevant national regulatory 241 authorities could be a first step in this direction (31).

242 Covid-19 could also provide the opportunity to explore new ways of collecting data from multiple 243 vaccine development programs. For instance, a public dataset with detailed information on the history 244 of regulatory review and approval status for every vaccine would offer significant social value by 245 facilitating research, shed light on cross-national differences in standards, procedures, and timelines; 246 and allow for the identification of bottlenecks - ultimately leading to improved vaccine access and 247 innovation.

248 The dataset could be maintained and hosted by an academic institution (in a similar fashion to the 249 Institute for Health Metrics and Evaluation at the University of Washington) or by a non250 governmental organization such as Gavi, the Vaccine Alliance. Ultimately, by facilitating research 251 that could accelerate regulatory processes and by providing vaccine manufacturers with more 252 certainty regarding prospective new markets, such a dataset could meaningfully contribute to greater 253 vaccination access and innovation.

254 While we are strongly convinced of the opportunities that are ahead of us, we also realize that the 255 approaches proposed in this article have several limitations. For instance, some of the technologies 256 described are not yet mature and have not yet proven their value. At the same time, since different regulatory agencies may have different approaches, some of the suggestions we make may be already accepted by some regulatory agencies while they may be useful suggestions for others. Also, we acknowledge that the numbers reported in Table 1, which show the full potential benefit of acceleration, were generated using very optimistic scenarios such as $100 \%$ coverage immediately after licensure; to date, this has happened only in very exceptional circumstances such as the implementation of the conjugate vaccines against meningococcus A and $\mathrm{C}$ in sub-Saharan Africa and the U.K., respectively.

264 In conclusion, the need to develop safe and effective Covid-19 vaccines in the shortest time possible, provides a unique opportunity to challenge the status quo and to introduce new tools such as smarter designs for clinical trials and new regulatory and health economic sciences. The tools suggest here and summarized in Figure 1 may be transformative and allow licensure of Covid-19 vaccines in 18 months from the beginning of vaccine development and reduce the time of normal vaccine development and recommendation to ten years or less. At the same time, adopting some or all of these recommendations would likely free up human and financial resources for $R \& D$ organizations to develop more and better vaccines. 


\section{Acknowledgements}

274 The authors wish to thank Daniel Cadarette, John Regan, and Nicola Groth for helpful comments and 275 discussions during the preparation of this paper and Giorgio Corsi for the artwork.

\section{Conflict of interest}

277 S.B. reports personal fees from GSK.

278 D.E.B. reports grants, travel grants, and/or personal fees from Bill and Melinda Gates Foundation, 279 IVI, GSK, Pfizer, Merck, Sanofi - outside the submitted work.

280 R.R. and S.P. are full-time employees of the GSK group of companies. GSK is a company that has a 281 direct financial interest in producing and marketing vaccines. 
Table 1. Estimated morbidity and mortality costs of delayed vaccine introduction ${ }^{\text {a }}$

\begin{tabular}{|c|c|c|c|}
\hline Vaccine & $\begin{array}{c}\text { Disease } \\
\text { deaths/year }\end{array}$ & Vaccine efficacy & $\begin{array}{c}\text { Potential maximum impact } \\
\text { on lives lost per year of } \\
\text { delay in vaccine } \\
\text { introduction }\end{array}$ \\
\hline $\begin{array}{l}\text { Malaria: } \\
\text { RTS,S in Sub-Saharan } \\
\text { Africa }\end{array}$ & $\begin{array}{c}\mathbf{4 0 7 , 0 0 0} \\
\text { deaths/year }\end{array}$ & $\begin{array}{c}\mathbf{5 7 . 7 \%} \\
(95 \% \text { CI: } 16.2-80.6)\end{array}$ & $\begin{array}{c}\mathbf{2 3 4 , 0 0 0} \\
(95 \% \text { CI: 65,934-328,042) }\end{array}$ \\
\hline $\begin{array}{l}\text { Human papillomavirus: } \\
\text { One dose vaccine } \\
\text { schedule in Sub-Saharan } \\
\text { Africa given current } \\
\text { supply constraints }\end{array}$ & $\begin{array}{l}\mathbf{8 1 , 6 8 7} \text { deaths/year } \\
\text { in a population of } \\
372,000 \text { women at } \\
\text { risk with } 119,284 \\
\text { new cases per year }\end{array}$ & $\begin{array}{c}\mathbf{9 5 . 8 9 \%} \\
(95 \% \text { CI: } 86-100) \\
\text { against vaccine strains }\end{array}$ & $\begin{array}{c}\mathbf{8 , ~ 6 6 2 ~}{ }^{1} \\
(95 \% \text { CI: } 7,768-9,032)\end{array}$ \\
\hline $\begin{array}{l}\text { Human papillomavirus: } \\
\text { Accelerated 2-dose } \\
\text { vaccine introduction in } \\
\text { LMICs }\end{array}$ & $\begin{array}{c}\mathbf{2 2 6 , 1 0 0} \\
\text { deaths/year in } \\
\text { LMICs }\end{array}$ & $\begin{array}{c}\mathbf{9 5 . 8 9 \%} \\
(95 \% \text { CI: } 86-100) \\
\text { against vaccine strains }\end{array}$ & $\begin{array}{c}\mathbf{1 5 1 , 6 8 7}^{2} \\
(95 \% \text { CI: } 136,112-158,270)\end{array}$ \\
\hline $\begin{array}{l}\text { Tuberculosis: } \\
\text { M72/AS01 } \\
\text { globally }\end{array}$ & $\begin{array}{l}\mathbf{1 , 7 0 0 , 0 0 0} \\
\text { deaths/year }\end{array}$ & $\begin{array}{c}\mathbf{5 4 . 0 \%} \\
(95 \% \text { CI: } 2.9-78.2)\end{array}$ & $\begin{array}{c}\mathbf{9 1 8 , 0 0 0} \\
(95 \% \text { CI: } 49,300-1,329,400)\end{array}$ \\
\hline
\end{tabular}

${ }^{\text {a }}$ To highlight the maximum potential impact of vaccination, the estimates report the theoretical lives per year that would be saved in the ideal situation of achieving $100 \%$ vaccination coverage immediately after licensure in all target geographies.

1 The UN estimates that the female population aged 10-14 in Sub-Saharan Africa is 67,248,900 (https://population.un.org/wpp/, accessed on May 6th, 2020). Given the mortality due to cervical cancer in Sub-Saharan Africa of 20/100,000 population and $70 \%$ coverage against cancer-causing strains and $95.5 \%$ vaccine efficacy against those strains, 8,991 is the number of additional deaths due to the extended use of a two-dose schedule given current capacity limits. HPV estimate based upon a global supply as of 2019 constrained to 30 million doses (WHO). Use of a one-dose schedule would double the population that could be vaccinated. Several studies indicate that effectiveness against long-term infection with HPV 16/18 is the same as a two-dose schedule (32). Currently, the program is capacitylimited to a one-year age group cohort with two doses rather than the five-year age group target population recommended by the WHO. Thus, by vaccinating with a one-dose schedule, vaccination could be expanded to two target cohorts. The mortality above represents the lives lost due to non-vaccination of this additional age group/year.

2 Estimation of global mortality rate of HPV from https://www.gavi.org/sites/default/files/board/minutes/2016/7dec/presentations/12\%20-\%20Review\%20of\%20Gavi\%20support\%20for\%20HPV\%20vaccine\%20presentation.pdf

(accessed on May 6th, 2020). Given that $85 \%$ of the deaths occur in LMICs and that the bivalent vaccine covers $70 \%$ of cancer-causing strains, the annual deaths averted would be 158,270 deaths/yr in LMICs. The study that modelled the data reported that the vaccine averted deaths over a ten-year period would be 2.4 million or 241,700/year (33). 


\section{References}

1. D. E. Bloom, V. Y. Fan, J. P. Sevilla, The broad socioeconomic benefits of vaccination. Sci Transl Med 10, (2018).

2. A. Hekele et al., Rapidly produced $\operatorname{SAM}((\mathrm{R}))$ vaccine against $\mathrm{H} 7 \mathrm{~N} 9$ influenza is immunogenic in mice. Emerg Microbes Infect 2, e52 (2013).

3. D. Wrapp et al., Cryo-EM structure of the 2019-nCoV spike in the prefusion conformation. Science 367, 1260-1263 (2020).

4. F. Y. Lin et al., Level of maternal IgG anti-group B streptococcus type III antibody correlated with protection of neonates against early-onset disease caused by this pathogen. $J$ Infect Dis 190, 928-934 (2004).

5. N. Eyal, M. Lipsitch, P. G. Smith, Human challenge studies to accelerate coronavirus vaccine licensure. J Infect Dis, (2020).

6. W. H. Chen et al., Single-dose Live Oral Cholera Vaccine CVD 103-HgR Protects Against Human Experimental Infection With Vibrio cholerae O1 E1 Tor. Clin Infect Dis 62, 1329-1335 (2016).

7. S. Li et al., Metabolic Phenotypes of Response to Vaccination in Humans. Cell 169, 862-877 e817 (2017).

8. T. D. Querec et al., Systems biology approach predicts immunogenicity of the yellow fever vaccine in humans. Nat Immunol 10, 116-125 (2009).

9. J. Weiner et al., Characterization of potential biomarkers of reactogenicity of licensed antiviral vaccines: randomized controlled clinical trials conducted by the BIOVACSAFE consortium. Sci Rep 9, 20362 (2019).

10. K. Kupferschmidt, Successful Ebola treatments promise to tame outbreak. Science 365, 628-629 (2019).

11. E. Andreano, A. Seubert, R. Rappuoli, Human monoclonal antibodies for discovery, therapy, and vaccine acceleration. Curr Opin Immunol 59, 130-134 (2019).

12. X. Chen et al., Human monoclonal antibodies block the binding of SARS-CoV-2 spike protein to angiotensin converting enzyme 2 receptor. Cell Mol Immunol, (2020).

13. F. Micoli et al., Comparative immunogenicity and efficacy of equivalent outer membrane vesicle and glycoconjugate vaccines against nontyphoidal Salmonella. Proc Natl Acad Sci U S A 115, 10428-10433 (2018).

14. N. Hernandez et al., Inherited IFNAR1 deficiency in otherwise healthy patients with adverse reaction to measles and yellow fever live vaccines. The Journal of experimental medicine 216, 20572070 (2019).

15. T. A. Lieu et al., Real-time vaccine safety surveillance for the early detection of adverse events. Med Care 45, S89-95 (2007).

16. A. M. Henao-Restrepo et al., Efficacy and effectiveness of an rVSV-vectored vaccine in preventing Ebola virus disease: final results from the Guinea ring vaccination, open-label, cluster-randomised trial (Ebola Ca Suffit!). Lancet 389, 505-518 (2017).

17. P. Balmer, R. Borrow, E. Miller, Impact of meningococcal C conjugate vaccine in the UK. J Med Microbiol 51, 717-722 (2002).

18. S. N. Ladhani et al., Vaccination of Infants with Meningococcal Group B Vaccine (4CMenB) in England. N Engl J Med 382, 309-317 (2020).

19. R. Rappuoli, S. Black, D. E. Bloom, Vaccines and global health: In search of a sustainable model for vaccine development and delivery. Sci Transl Med 11, eaaw2888 (2019).

20. A. K. Rowe et al., The burden of malaria mortality among African children in the year 2000. Int J Epidemiol 35, 691-704 (2006).

21. P. L. Alonso et al., Efficacy of the RTS,S/AS02A vaccine against Plasmodium falciparum infection and disease in young African children: randomised controlled trial. Lancet 364, 1411-1420 (2004).

22. Gavi the Vaccine Alliance, Vaccine Investment Strategy, Programme \& Policy Committee Meeting, 18-19 October 2018. Appendix 3: Malaria Vaccine. https://www.gavi.org/sites/default/files/document/ppc-meeting-18-19-october-2018---vis-appendix3--malaria-vaccine-analysispdf.pdf.

23. L. Bruni et al., ICO/IARC Information Centre on HPV and Cancer (HPV Information Centre). Human Papillomavirus and Related Diseases in Africa. Summary Report 17 June 2019. 
24. D. M. Harper et al., Efficacy of a bivalent L1 virus-like particle vaccine in prevention of infection with human papillomavirus types 16 and 18 in young women: a randomised controlled trial. Lancet 364, 1757-1765 (2004).

25. H. G. Eichler et al., The risks of risk aversion in drug regulation. Nat Rev Drug Discov 12, 907-916 (2013).

26. J. J. Darrow, J. Avorn, A. S. Kesselheim, FDA Approval and Regulation of Pharmaceuticals, 19832018. JAMA 323, 164-176 (2020).

27. J. M. Sharfstein, Reform at the FDA-In Need of Reform. JAMA 323, 123-124 (2020).

28. U.S. Food and Drug Administration (FDA), Guidance document: Adaptive Design Clinical Trials for Drugs and Biologics. November 2019. https://www.fda.gov/regulatory-information/search-fdaguidance-documents/adaptive-design-clinical-trials-drugs-and-biologics-guidance-industry.

29. U.S. Food and Drug Administration (FDA), Guidance document: Biomarker Qualification: Evidentiary Framework. December 2018. https://www.fda.gov/regulatory-information/search-fdaguidance-documents/biomarker-qualification-evidentiary-framework.

30. U.S. Food and Drug Administration (FDA), Guidance document: Real World Data / Real World Evidence RWD/RWE. July 2018. https://www.fda.gov/regulatory-information/search-fda-guidancedocuments/real-world-data-real-world-evidence-rwdrwe.

31. U.S. Food and Drug Administration (FDA), Master Protocols: Efficient Clinical Trial Design Strategies To Expedite Development of Oncology Drugs and Biologics. October 2018. https://www.fda.gov/regulatory-information/search-fda-guidance-documents/master-protocolsefficient-clinical-trial-design-strategies-expedite-development-oncology-drugs-and.

32. A. R. Kreimer et al., Proof-of-principle evaluation of the efficacy of fewer than three doses of a bivalent HPV16/18 vaccine. J Natl Cancer Inst 103, 1444-1451 (2011).

33. N. G. Campos et al., The health and economic impact of scaling cervical cancer prevention in 50 low- and lower-middle-income countries. Int J Gynaecol Obstet 138 Suppl 1, 47-56 (2017). 